\title{
The Diversity of Massive Star Outbursts. I. Observations of Sn2009ip, Ugc 2773 Ot2009-1, and Their Progenitors
}

\section{Citation}

Foley, Ryan J., Edo Berger, Ori Fox, Emily M. Levesque, Peter J. Challis, Inese I. Ivans, James E. Rhoads, and Alicia M. Soderberg. 2011. "THE DIVERSITY OF MASSIVE STAR OUTBURSTS. I. OBSERVATIONS OF SN2009ip, UGC 2773 OT2009-1, AND THEIR PROGENITORS." The Astrophysical Journal 732 (1): 32. https://doi.org/10.1088/0004-637x/732/1/32.

\section{Permanent link}

http://nrs.harvard.edu/urn-3:HUL.InstRepos:41397443

\section{Terms of Use}

This article was downloaded from Harvard University's DASH repository, and is made available under the terms and conditions applicable to Open Access Policy Articles, as set forth at http:// nrs.harvard.edu/urn-3:HUL.InstRepos:dash.current.terms-of-use\#OAP

\section{Share Your Story}

The Harvard community has made this article openly available.

Please share how this access benefits you. Submit a story.

\section{Accessibility}


Draft Version April 15, 2018

Preprint typeset using $\mathrm{LAT}_{\mathrm{E}} \mathrm{X}$ style emulateapj v. 11/10/09

\title{
THE DIVERSITY OF MASSIVE STAR OUTBURSTS I: OBSERVATIONS OF SN 2009ip, UGC 2773 OT2009-1, AND THEIR PROGENITORS
}

\author{
Ryan J. Foley ${ }^{1,2}$, Edo Berger ${ }^{1}$, Ori Fox ${ }^{3}$, Emily M. Levesque ${ }^{4,5}$, Peter J. Challis ${ }^{1}$, Inese I. Ivans ${ }^{6}$, \\ James E. Rhoads ${ }^{7}$, Alicia M. Soderberg ${ }^{1}$ \\ Draft version April 15, 2018
}

\begin{abstract}
Despite both being outbursts of luminous blue variables (LBVs), SN 2009ip and UGC 2773 OT20091 have very different progenitors, spectra, circumstellar environments, and possibly physical mechanisms that generated the outbursts. From pre-eruption HST images, we determine that SN 2009ip and UGC 2773 OT2009-1 have initial masses of $\gtrsim 60$ and $\gtrsim 25 M_{\odot}$, respectively. Optical spectroscopy shows that at peak SN 2009ip had a 10,000 K photosphere and its spectrum was dominated by narrow H Balmer emission, similar to classical LBV giant outbursts, also known as "supernova impostors." The spectra of UGC 2773 OT2009-1, which also have narrow H $\alpha$ emission, are dominated by a forest of absorption lines, similar to an F-type supergiant. Blueshifted absorption lines corresponding to ejecta at a velocity of $2000-7000 \mathrm{~km} \mathrm{~s}^{-1}$ are present in later spectra of SN 2009ip — an unprecedented observation for LBV outbursts, indicating that the event was the result of a supersonic explosion, rather than a subsonic outburst. The velocity of the absorption lines increases between two epochs, suggesting that there were two explosions in rapid succession. A rapid fading and rebrightening event concurrent with the onset of the high-velocity absorption lines is consistent with the double-explosion model. A near-infrared excess is present in the spectra and photometry of UGC 2773 OT2009-1 that is consistent with $\sim 2100 \mathrm{~K}$ dust emission. We compare the properties of these two events and place them in the context of other known massive star outbursts such as $\eta$ Car, NGC 300 OT2008-1, and SN 2008S. This qualitative analysis suggests that massive star outbursts have many physical differences which can manifest as the different observables seen in these two interesting objects.

Subject headings: circumstellar matter — stars: evolution — stars: individual (UGC 2773 OT2009-1, SN 2009ip) — stars: mass loss — stars: variable: other — stars: winds, outflows - supernovae: general
\end{abstract}

\section{INTRODUCTION}

Very massive stars appear to go through a phase of instability and large mass loss; during this stage, a star is a member of the luminous blue variable (LBV) class (see Humphreys \& Davidson 1994 for a review). In addition to low-amplitude variability (called S Dor variability after the prototypical LBV), where the star ejects mass from its envelope but its bolometric luminosity remains nearly constant, some LBVs have "giant eruptions." Giant eruptions can expel $\gtrsim 1 M_{\odot}$ of material, while having a luminosity similar to the lowest-luminosity supernovae (SNe). The classical examples of giant eruptions are $\mathrm{P}$ Cygni in 1600 and $\eta$ Car in 1843, and more recent, extragalactic examples include SN 1961V (Goodrich et al. 1989; Filippenko et al. 1995; Van Dyk et al. 2002; but see Chu et al. 2004), V12/SN 1954J (Smith et al. 2001; Van Dyk et al. 2005), SN 1997bs (Van Dyk et al. 2000), SN 2000ch (Wagner et al. 2004), and V37/SN 2002kg (Weis \& Bomans 2005; Maund et al.

1 Harvard-Smithsonian Center for Astrophysics, 60 Garden Street, Cambridge, MA 02138, USA.

2 Clay Fellow. Electronic address rfoley@cfa.harvard.edu .

3 Department of Astronomy, University of Virginia, P.O. Box 400325, Charlottesville, VA 22904, USA.

4 Institute for Astronomy, University of Hawaii, 2680 Woodlawn Dr., Honolulu, HI 96822, USA.

5 Predoctoral Fellow, Smithsonian Astrophysical Observatory

6 Department of Physics and Astronomy, University of Utah, Salt Lake City, UT 84112, USA.

7 School of Earth and Space Exploration, Arizona State University, P.O. Box 871404, Tempe, AZ 85287, USA
2006; Van Dyk et al. 2006). Other potential examples exist, but all events listed above have pre-event imaging where the progenitor star has been identified as a probable or definite LBV.

Two examples of a new class of stellar eruptions have recently emerged. The progenitors of NGC 300 OT20081 and SN 2008S were both detected in pre-event Spitzer images, but were undetected to deep limits in the optical (Prieto et al. 2008; Berger et al. 2009b; Bond et al. 2009), indicating significant reddening from circumstellar dust. The progenitor stars were originally believed to have ZAMS masses of $8-20 M_{\odot}$, which is below that of the least massive LBVs. Using the stars in the vicinity of NGC 300 OT2008-1, Gogarten et al. (2009) found a slightly higher mass range of $12-25 M_{\odot}$. A reasonable range for the initial mass of the progenitors is $\sim 10$ - 25M $M_{\odot}$, with NGC 300 OT2008-1, having a more luminous progenitor, being toward the upper end of that range.

Recently, two transients were discovered with one being very similar to classical LBV giant eruptions (SN 2009ip), and another sharing characteristics of both LBV giant eruptions and the outbursts of the dusty stars discussed above (UGC 2773 OT2009-1). SN 2009ip was discovered by Maza et al. (2009) in NGC 7259 ( $\mu=$ $32.05 \pm 0.15 \mathrm{mag} 8 ; \quad D \approx 24 \mathrm{Mpc}$ ) on 2009 August 26 (UT

8 We use the distance modulus corresponding to the Hubble distance for NGC 7259 with $H_{0}=73 \mathrm{~km} \mathrm{~s}^{-1} \mathrm{Mpc}^{-1}$ and correcting for the Virgo Infall and Great Attractor flow model of Mould et al. 
dates will be used throughout this paper). Miller et al. (2009) noted that SN 2009ip had been variable for several years and identified a possible progenitor with $M_{\mathrm{F} 606 \mathrm{~W}} \approx$ -10.1 mag, in an archival Hubble Space Telescope (HST ) image. The variability and high luminosity of the event led Miller et al. (2009) to suggest that SN 2009ip was either an LBV or cataclysmic variable outburst. An early spectrum of the event showed a blue continuum with relatively narrow (FWHM $=550 \mathrm{kms}^{-1}$ ) $\mathrm{H}$ Balmer lines. The combination of the spectrum with the relatively low absolute magnitude $(R \approx-13.7 \mathrm{mag})$ led us to conclude that SN 2009ip was an LBV giant eruption (Berger, Folev, \& Ivans 2009a). The transient underwent extreme variability shortly after maximum 9 , fading by at least $3 \mathrm{mag}$ in 16 days and rebrightening by $2 \mathrm{mag}$ in the next 10 days (Li et al. 2009), reminiscent of the variability immediately before maximum of the 1843 eruption of $\eta$ Car (Frew 2004) and immediately after maximum in the LBV outburst SN 2000ch (Wagner et al. 2004). Smith et al. (2009b, hereafter S09) presented a historical light curve of SN 2009ip that begins 5 years before maximum light, excluding the $H S T$ image of the progenitor. The star varied by at least $1.5 \mathrm{mag}$ during this time. S09 presented additional data which led them to conclude that SN 2009ip was the giant eruption of a $50-80 M_{\odot} \mathrm{LBV}$.

UGC 2773 OT2009-1 was discovered by Boles (2009) in UGC $2773(\mu=28.82 \pm 0.17 \mathrm{mag} ; D \approx 6 \mathrm{Mpc})$ on 2009 August 18. It was originally reported as a possible SN. We obtained a spectrum and noted that it had a peculiar spectrum with relatively narrow $\left(\mathrm{FWHM}=350 \mathrm{~km} \mathrm{~s}^{-1}\right)$ $\mathrm{H} \alpha$ emission, P-Cygni lines from the Ca II NIR triplet, and [Ca II] emission lines (Berger \& Foley 2009). We also noted that the spectrum was similar to that of NGC 300 OT2008-1 and mentioned that it was possibly a very low-luminosity SN II or an LBV outburst, "but the strong [Ca II] emission would be unexpected in this case." Berger \& Folev (2009) also detected a potential progenitor star in archival HST images. S09 presented a historical light curve of SN 2009ip that begins 9 years (excluding the HST image of the progenitor) before maximum light. The star slowly increased from $\mathrm{F} 814 \mathrm{~W}=22.22 \mathrm{mag}$ to an unfiltered magnitude of 17.70 mag at maximum light, corresponding to a linear increase of $\sim 0.4$ mag year ${ }^{-1}$ before outburst. S09 concluded from the progenitor identification, their historic light curve, the peak luminosity, and optical light curves that UGC 2773 OT2009-1 was the outburst of a $\gtrsim 20 M_{\odot}$ LBV.

This is the first in a series of papers where we investigate the diversity of massive star outbursts. In this paper we demonstrate the heterogeneity of the class with observations of SN 2009ip and UGC 2773 OT2009-1. In future papers, we will detail the properties of the class and the links between observations and the physical mecha-

(2000). Smith et al. (2009b) use a distance modulus of $31.55 \mathrm{mag}$, which differs by $0.50 \mathrm{mag}$ from our assumed value, corresponding to the Hubble-flow distance modulus correcting only for the CMB dipole.

9 To be consistent with S09, we adopt MJD $=55061.5$ and MJD $=55071.75$ as times of maximum light for UGC 2773 OT2009-1 and SN 2009ip, respectively. However, we note that the objects may reach their true maximum later, which UGC 2773 OT2009-1 already has (as shown by data presented in S09) nisms which cause the outbursts. In Section 2, we present ultraviolet (UV), optical, and near-infrared (NIR) photometry and optical and NIR spectroscopy of UGC 2773 OT2009-1 and SN 2009ip. In this section, we also refine previous identifications of the progenitors. In Section 3. we examine the progenitor masses, the spectroscopic characteristics of the outbursts, and the spectralenergy distributions (SEDs) of the events. In Section 4 , we discuss how these outbursts connect to previous massive star outbursts and the mass loss history and ultimate fates of massive stars. We summarize our conclusions in Section 5 .

\section{OBSERVATIONS AND DATA REDUCTION}

\subsection{Identification and HST Photometry of the Progenitors}

UGC 2773 was observed with HST/WFPC2 on 1999 August 14 (Program 8192; PI Seitzer). The observations included two exposures of $600 \mathrm{~s}$ each with the F606W and F814W (roughly $V$ and $I$ ) filters. NGC 7259 was observed with $H S T$ /WFPC2 on 1999 June 29 (Program 6359; PI Stiavelli). Exposures of 200 and $400 \mathrm{~s}$ were obtained with the F606W filter.

To determine whether the progenitors of SN 2009ip and UGC 2773 OT2009-1 are detected in the archival $H S T$ observations, we performed differential astrometry using optical observations of the transients. Observations of UGC 2773 OT2009-1 were obtained with the Gemini Multi-Object Spectrograph (GMOS) on the GeminiNorth 8-m telescope, and the astrometry was performed using 55 objects in common with the $H S T / W F P C 2$ images resulting in an astrometric rms of $\sigma_{\mathrm{GB} \rightarrow \mathrm{HST}}=$ 24 mas in each coordinate. Observations of SN 2009ip were obtained with the Inamori Magellan Areal Camera and Spectrograph (IMACS) on the Magellan/Baade 6.5$\mathrm{m}$ telescope, and the astrometry was performed using 10 objects in common with the $H S T$ /WFPC2 image resulting in an astrometric rms of $\sigma_{\mathrm{GB} \rightarrow \mathrm{HST}}=38$ mas in each coordinate.

The positions of the two transients on the archival $H S T$ images are shown in Figure 11. In both cases, we find a clear coincidence with objects in the archival HST images. For SN 2009ip we find an offset of $24 \pm 38$ mas relative to the object in the WFPC2/F606W image, while for UGC 2773 OT2009-1 we find an offset of $32 \pm 24$ mas relative to the object in the $\mathrm{WFPC} 2 / \mathrm{F} 606 \mathrm{~W}$ and $\mathrm{F} 814 \mathrm{~W}$ images.

The measurements of the photometry for UGC 2773 OT2009-1 and nearby stars were done using HSTphot 1.1 (Dolphin 2000). HSTphot was run using a weighted PSFfit, which is recommended for crowded fields, and a local sky determination, which is recommended for rapidly varying backgrounds. HSTphot performs the conversion from HST/WFPC2 flight magnitudes to the Bessel magnitude system. Our astrometry and photometry for the nominal progenitor of UGC 2773 OT2009-1 and nearby stars are listed in Table 1 .

We performed photometry of the point source coincident with SN 2009ip using a $0.5^{\prime \prime}$ aperture and a zeropoint of $22.47 \mathrm{mag}$ appropriate for the F606W filter. We further applied a correction of $-0.29 \mathrm{mag}$ to convert to the Vega system, and applied a correction for the Galactic extinction of $0.05 \mathrm{mag}$. The resulting magnitude of 

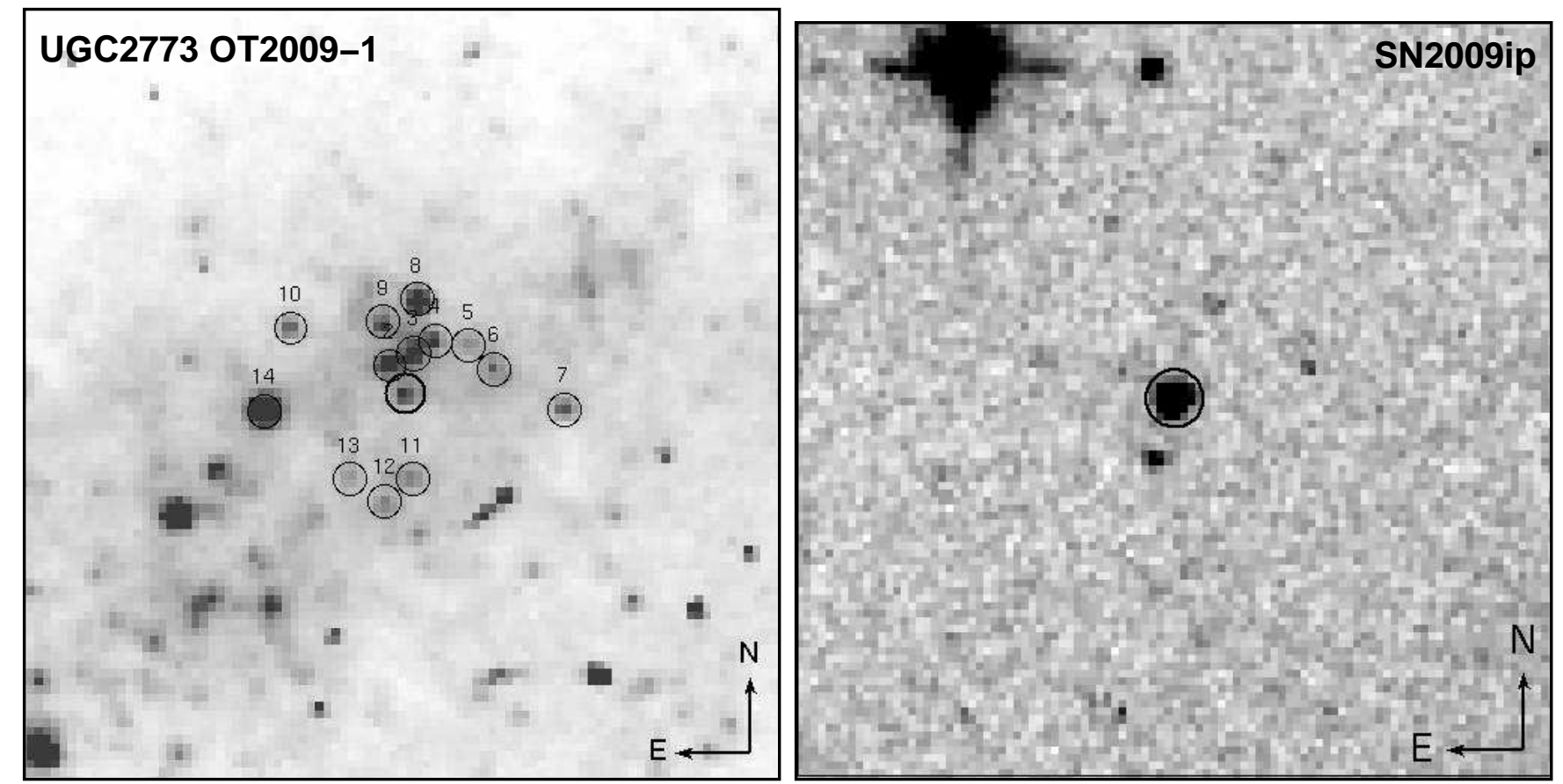

Figure 1. HST/WFPC2 F606W image at the position of UGC 2773 OT2009-1 (left) and SN 2009ip (right) obtained 10 years before maximum. Both images are $10^{\prime \prime} \times 10^{\prime \prime}$, and North is up and East is left. The UGC 2773 OT2009-1 and SN 2009ip images have pixel scales of $0.1^{\prime \prime}$ pixel $^{-1}$. The position of each transient is marked by the black circle whose radius corresponds to $10 \sigma$ uncertainty in the position.

Table 1

HST Photometry of Stars Near UGC 2773 OT2009-1

\begin{tabular}{ccccc}
\hline \hline Object & R.A. & Dec. & F606W (mag) & F814W (mag) \\
\hline $1^{\text {a }}$ & $03: 32: 7.240$ & $+47: 47: 39.60$ & $22.824(0.032)$ & $22.286(0.053)$ \\
2 & $03: 32: 7.258$ & $+47: 47: 39.99$ & $22.474(0.027)$ & $22.164(0.050)$ \\
3 & $03: 32: 7.229$ & $+47: 47: 40.14$ & $22.672(0.036)$ & $22.222(0.054)$ \\
4 & $03: 32: 7.203$ & $+47: 47: 40.29$ & $22.853(0.035)$ & $22.591(0.081)$ \\
5 & $03: 32: 7.162$ & $+47: 47: 40.23$ & $23.864(0.072)$ & $22.729(0.074)$ \\
6 & $03: 32: 7.132$ & $+47: 47: 39.94$ & $23.413(0.051)$ & $22.748(0.092)$ \\
7 & $03: 32: 7.048$ & $+47: 47: 39.45$ & $22.925(0.034)$ & $20.956(0.020)$ \\
8 & $03: 32: 7.225$ & $+47: 47: 40.79$ & $21.724(0.016)$ & $21.043(0.022)$ \\
9 & $03: 32: 7.266$ & $+47: 47: 40.51$ & $23.007(0.039)$ & $22.548(0.067)$ \\
10 & $03: 32: 7.377$ & $+47: 47: 40.44$ & $22.967(0.035)$ & $21.682(0.035)$ \\
11 & $03: 32: 7.230$ & $+47: 47: 38.60$ & $23.762(0.092)$ & $23.182(0.113)$ \\
12 & $03: 32: 7.263$ & $+47: 47: 38.34$ & $23.578(0.058)$ & $23.095(0.100)$ \\
13 & $03: 32: 7.306$ & $+47: 47: 38.61$ & $24.015(0.081)$ & $23.304(0.116)$ \\
14 & $03: 32: 7.409$ & $+47: 47: 39.44$ & $21.194(0.026)$ & $19.934(0.017)$ \\
\hline
\end{tabular}

a Star 1 is identified as the progenitor of UGC 2773 OT2009-1.

the source is $21.84 \pm 0.25$ mag. For reasonable colors (see Section 3.1.1], this corresponds to $M_{V}=-10.3 \mathrm{mag}$.

\subsection{Ultraviolet, Optical, and Near-Infrared Photometry}

We obtained optical photometry of UGC 2773 OT2009-1 with the Gemini Multi-Object Spectrograph (GMOS) on the Gemini-North 8-m telescope in the gri filters. We performed the photometry using IRAF/phot with the standard GMOS zero-point: $\square$. Our results are presented in Table 2.

We obtained near-infrared (NIR) photometry of UGC 2773 OT2009-1 with FanCam, a $1024 \times 1024$ HAWAII-I HgCdTe imaging system on the University of Virginia's 31-inch telescope at Fan Mountain, just

\footnotetext{
10 http://www.gemini.edu/sciops/instruments/gmos/imaging
}

outside of Charlottesville, VA (Kanneganti et al. 2009). Each epoch consists of fifteen minutes of integration in $J H K_{s}$ bands, which have detection limits at the $10 \sigma$ level of $0.066,0.098$, and $0.156 \mathrm{mJy}$ (or 18.5, 17.5, and $16.5 \mathrm{mag}$ ), respectively. Individual exposures are skybackground limited and have an integration time of either 30 or $60 \mathrm{~s}$. Flat-field frames are composed of dusk and dawn sky observations. We employed standard NIR data reduction techniques in IRAFM. Because of the relatively small galaxy size, it was possible to fit the entire galaxy in a single array quadrant. Empty quadrants were efficiently utilized as sky exposures. Data

11 IRAF is distributed by the National Optical Astronomy Observatory, which is operated by the Association of Universities for Research in Astronomy (AURA) under cooperative agreement with the National Science Foundation. 
Table 2

UV and Optical Photometry of UGC 2773 OT2009-1 and SN 2009ip

\begin{tabular}{rcccc}
\hline \hline Object & MJD & Filter & Mag & Telescope \\
\hline UGC 2773 OT2009-1 & 51404.13 & F606W & $22.82(0.03)$ & $H S T$ \\
UGC 2773 OT2009-1 & 51404.14 & F814W & $22.29(0.05)$ & HST \\
UGC 2773 OT2009-1 & 55078.38 & $J$ & $15.47(0.06)$ & Fan Mountain \\
UGC 2773 OT2009-1 & 55078.40 & $H$ & $14.99(0.06)$ & Fan Mountain \\
UGC 2773 OT2009-1 & 55078.39 & $K_{s}$ & $14.63(0.07)$ & Fan Mountain \\
UGC 2773 OT2009-1 & 55078.53 & $g$ & $18.32(0.01)$ & Gemini-North \\
UGC 2773 OT2009-1 & 55078.53 & $r$ & $17.22(0.01)$ & Gemini-North \\
UGC 2773 OT2009-1 & 55078.53 & $i$ & $16.68(0.01)$ & Gemini-North \\
UGC 2773 OT2009-1 & 55089.31 & $J$ & $15.47(0.06)$ & Fan Mountain \\
UGC 2773 OT2009-1 & 55089.32 & $H$ & $14.91(0.07)$ & Fan Mountain \\
UGC 2773 OT2009-1 & 55089.33 & $K_{s}$ & $14.91(0.09)$ & Fan Mountain \\
\hline SN 2009ip & 51358.50 & F606W & $21.84(0.17)$ & HST \\
SN 2009ip & 55084.44 & UVW2 & $21.09(0.19)$ & Swift \\
SN 2009ip & 55084.45 & UVM2 & $20.92(0.28)$ & Swift \\
SN 2009ip & 55084.44 & UVW1 & $20.69(0.18)$ & Swift \\
SN 2009ip & 55084.44 & $U$ & $20.29(0.16)$ & Swift \\
SN 2009ip & 55084.44 & $B$ & $20.64(0.09)$ & Swift \\
SN 2009ip & 55084.45 & $V$ & $20.47(0.37)$ & Swift \\
\hline
\end{tabular}

were taken with the galaxy placed in each quadrant and each quadrant was reduced separately. Ultimately, all reduced quadrants were coadded. We performed photometry with IRAF's PSF package. For magnitude calibration, the transient is compared to 2MASS reference stars located in the field of view. Table 2 lists our $J H K_{s}$ photometry, which is similar to the single epoch $J H K_{s}$ data from S09.

We obtained UV and optical observations of SN 2009ip with the Swift UV/optical telescope on 2009 September 10. The data were processed using standard routines within the HEASOFT package. Photometry of the transient in all filters, with the exception of UVW2, was performed using a $2^{\prime \prime}$ aperture to avoid contamination from nearby objects. Aperture corrections to the standard $5^{\prime \prime}$ aperture were determined using isolated stars; photometry of the source in the UVW2 filter was performed using a $5^{\prime \prime}$ aperture.

\subsection{X-ray Observations}

We observed SN 2009ip and UGC 2773 OT2009-1 with the Swift X-ray Telescope on 2009 September 10 for a total exposure time of 9.0 and $4.2 \mathrm{ks}$, respectively. No X-ray counterpart is detected at the position of either source to a limit of $F_{X} \lesssim 2.8 \times 10^{-14}$ and $\lesssim 1.1 \times 10^{-13} \mathrm{erg} \mathrm{s}^{-1} \mathrm{~cm}^{-2}$, respectively (95\% limit). In both cases we assume a power law model with an electron index of -2 , and account for the Galactic neutral hydrogen column. The corresponding limits on the luminosity are $L_{X} \lesssim 1.9 \times 10^{39}$ and $\lesssim 4.8 \times 10^{38} \mathrm{erg} \mathrm{s}^{-1}$. These limits are comparable to the X-ray emission from SNe on a similar timescale (e.g., Soderberg et al. 2008).

\subsection{Radio Observations}

We observed the both LBV candidates with the Very Large Array ${ }^{12}$ (VLA) following their optical discovery to search for radio counterparts, under Rapid Response programs AS1001 and AS1002 (PI Soderberg). Our radio observations were carried out at two frequencies, 8.46

\footnotetext{
12 The Very Large Array is operated by the National Radio Astronomy Observatory, a facility of the National Science Foundation operated under cooperative agreement by Associated Universities, Inc.
}

Table 3

VLA observations of UGC 2773 OT2009-1 and SN 2009ip

\begin{tabular}{lccc}
\hline \hline Object & $\begin{array}{c}\text { Date } \\
(\mathrm{UT})\end{array}$ & $\begin{array}{c}F_{\nu, 8.5} \\
(\mu \mathrm{Jy})\end{array}$ & $\begin{array}{c}F_{\nu, 22} \\
(\mu \mathrm{Jy})\end{array}$ \\
\hline SN 2009ip & 2009 Sep 7.36 & $\ldots$ & $-122 \pm 243$ \\
$\ldots$ & 2009 Sep 9.24 & $67 \pm 47$ & $\ldots$ \\
UGC 2773 OT2009-1 & 2009 Sep 13.57 & $\ldots$ & $-57 \pm 66$ \\
$\ldots$ & 2009 Sep 16.51 & $11 \pm 27$ & $\cdots$ \\
\hline
\end{tabular}

Note. - Uncertainties are $1 \sigma$ rms map noise.

and $22.5 \mathrm{GHz}$, on dates spanning 2009 September 7.36 - 16.51 in the C-array antenna configuration. All observations were taken in standard continuum observing mode with a bandwidth of $2 \times 50 \mathrm{MHz}$. Phase referencing was performed with calibrators J0325+469 and J2213254, and we used 3C38 (J0137+331) for flux calibration. Data were reduced using standard packages within the Astronomical Image Processing System (AIPS).

We detect no radio sources in positional coincidence with either object and derive upper limits summarized in Table [3. At $8.5 \mathrm{GHz}$, our upper limit:13 correspond to $L_{\nu}<1.3 \times 10^{26} \mathrm{erg} \mathrm{s}^{-1} \mathrm{~Hz}^{-1}$ and $L_{\nu}<$ $2.6 \times 10^{24} \mathrm{erg} \mathrm{s}^{-1} \mathrm{~Hz}^{-1}$ for SN 2009ip and UGC 2773 OT2009-1, respectively. These limits are less luminous than an extrapolation of the observed SN 1961V radio emission at $t \approx 10$ years, to a similarly early epoch as $L_{\nu} \propto t^{-1.75}$ (Stockdale et al. 2001). We note, however, that the SN $1961 \mathrm{~V}$ radio emission may have reached maximum intensity significantly later than our observations of UGC 2773 OT2009-1 and SN 2009ip, similar to the radio evolution of SNe IIn which typically reach maximum light several years after the explosion (van Dyk et al. 1996).

A comparison of these radio upper limits for the outbursts to the observed properties of other core-collapse SNe places them among the least luminous events, 2-4 orders of magnitude less luminous than the most powerful SNe IIn, and 4-200 times higher than the early radio signal seen for SN 1987A (Ball et al. 1995). Through this

13 Upper limits are calculated as the measured flux density at the optical position summed with $2 \times \mathrm{rms}$ the off-source map noise. 
simple comparison we emphasize that radio data alone cannot distinguish between massive star outbursts and catastrophic explosions. However, with the 10-fold increase in continuum sensitivity provided by the EVLA we will begin to map out the radio properties for massive star outbursts and enable direct comparisons with those of core-collapse SNe (e.g., NRAO Key Project AS1020, "Exotic Explosions,Eruptions,and Disruptions: A New Transient Phase-Space", PI Soderberg).

\subsection{Optical Spectroscopy}

We obtained low- and medium-resolution spectra of SN 2009ip and UGC 2773 OT2009-1 with the MagE spectrograph (Marshall et al. 2008) on the Magellan Clay $6.5 \mathrm{~m}$ telescope, the Blue Channel spectrograph (Schmidt, Weymann, \& Foltz 1989) on the MMT $6.5 \mathrm{~m}$ telescope, and GMOS (Hook et al. 2004) on the GeminiNorth $8 \mathrm{~m}$ telescope. A journal of our optical spectroscopic observations can be found in Table 4 .

Standard CCD processing and spectrum extraction were accomplished with IRAF. The data were extracted using the optimal algorithm of Horne (1986). Loworder polynomial fits to calibration-lamp spectra were used to establish the wavelength scale, and small adjustments derived from night-sky lines in the object frames were applied. For the MagE spectra, the sky was subtracted from the images using the method described by Kelson (2003). The GMOS data were reduced using the Gemini IRAF package (for details, see Foley et al. 2006). We employed our own IDL routines to flux calibrate the data and remove telluric lines using the wellexposed continua of the spectrophotometric standards (Wade \& Horne 1988; Foley et al. 2003, 2009).

Representative spectra of SN 2009ip and UGC 2773 OT2009-1 are presented in Figure 2, Both objects have similar blue continua, but the line features are very different. SN 2009ip has few line features besides strong $\mathrm{H}$ Balmer lines, Na D, and He I. Although UGC 2773 OT2009-1 has a strong $\mathrm{H} \alpha$ line, it is much narrower and weaker than that of SN 2009ip. UGC 2773 OT2009-1 also displays many additional narrow line features, including lines from intermediate mass and Fe-group elements. Blueward of $~ 5500 \AA$, UGC 2773 OT2009-1 is dominated by a forest of Fe II lines. Finally, UGC 2773 OT2009-1 has very strong Ca II NIR triplet lines and [Ca II] $\lambda \lambda 7291,7324$ lines. These features, and particularly $[\mathrm{Ca}$ II] are rarely seen in classical LBV outbursts, but were distinguishing features of SN 1999bw (Garnavich et al. 1999), SN 2008S (e.g., Smith et al. 2008b), and NGC 300 OT2008-1 (e.g., Berger et al. 2009b).

The spectra from 2009 September 21 (corresponding to days 34 and 24 for UGC 2773 OT2009-1 and SN 2009ip, respectively) were obtained on the same night as the LRIS spectra shown by $\underline{\text { S09. }}$.

\subsection{Near-Infrared Spectroscopy}

On 2009 September 9 (22 days after maximum), we obtained a 2400 s NIR spectrum of UGC 2773 OT2009-1 with TripleSpec, a medium resolution NIR spectrograph located at Apache Point Observatory. This spectrograph is one of three NIR, cross-dispersed spectrographs covering wavelengths from $1-2.4 \mu \mathrm{m}$ simultaneously at a resolution of 3500 (Wilson et al. 2004; Herter et al.|2008).
We collected eight, 300-s sky-background limited exposures, for a total integration time of $2400 \mathrm{~s}$. We extracted the spectrum with a modified version of the IDL-based SpexTool (Cushing. Vacca, \& Ravner 2004). This tool removes any contribution from the underlying galactic arm by fitting the background with a 2nd order polynomial.

\section{RESULTS}

\subsection{Progenitor Masses}

\subsubsection{SN 2009ip}

We use the absolute magnitude, $M_{V}=-10.3 \mathrm{mag}$, of the progenitor of SN 2009ip, along with an estimated range of $V-I$ colors of -0.05 to $1.4 \mathrm{mag}$ (representative of LBV colors spanning from $\mathrm{O}$ to $\mathrm{F}$ spectral types), to plot it as a line on a color-magnitude diagram (Figure 3). For this progenitor we find a Milky Way extinction of $E(B-V)=0.019 \mathrm{mag}\left(A_{V}=0.05 \mathrm{mag}\right.$; Schlegel, Finkbeiner, \& Davis 1998). We adopt a distance modulus of $\mu=32.05 \mathrm{mag}$ for NGC 7259 (see discussion in Section 1), and assume no additional host galaxy or circumstellar extinction.

In Figure 3, we compare the color of the SN 2009ip progenitor to the non-rotating, standard mass-loss evolutionary tracks of the Geneva group (Schaller et al. 1992). From this plot we can place a lower initial mass limit of $60 M_{\odot}$ on the progenitor of SN 2009ip in the absence of a color estimate for this progenitor, the higher-mass evolutionary tracks all coincide with its estimated location on the color-magnitude diagram, precluding us from placing an upper limit on this initial mass estimate. Figure 3 assumes a solar metallicity for these tracks; however, we find that our progenitor mass prediction is consistent across the full range of metallicities accommodated by the Geneva evolutionary tracks $\left(Z=0.05 Z_{\odot}\right.$ to $\left.Z=2 Z_{\odot}\right)$. It should be noted that an increased amount of extinction, from the host galaxy or circumstellar environment, could also effectively increase the estimated initial mass of this progenitor.

S09 estimated the initial mass for the progenitor of SN 2009ip to be $50-80 M_{\odot}$. Although the HST photometry from S09 is the same as that presented here, their assumed distance modulus is $0.50 \mathrm{mag}$ smaller than our assumed value. They also make no color correction to transform the $\mathrm{F} 606 \mathrm{~W}$ measurements into $V$. Despite these differences, the two mass ranges are similar.

\subsubsection{UGC 27r3 OT2009-1}

Using its $M_{V}$ and $V-I$ color, we are able to determine an estimate of the initial mass for the progenitor of UGC 2773 OT2009-1 (Figure 3). There is significant Milky Way extinction of $E(B-V)=0.564 \mathrm{mag}\left(A_{V}=\right.$ 1.75 mag; Schlegel, Finkbeiner, \& Davis 1998), which we convert to $E(V-I)=0.902 \mathrm{mag}$ (Schultz \& Wiemer 1975). We use a distance modulus of $\mu=28.82 \mathrm{mag}$ and initially assume no host galaxy or circumstellar extinction.

From Figure 3, we find that the progenitor of UGC 2773 OT2009-1 is consistent with an initial mass of $\sim 20 M_{\odot}$. Our progenitor mass prediction remains the same across the full range of metallicities covered by the Geneva evolutionary tracks, and is consistent with the value found by $\mathbf{S 0 9}$. 
Table 4

Log of Optical Spectral Observations

\begin{tabular}{|c|c|c|c|c|c|}
\hline Phase $^{\mathrm{a}}$ & UT Date & $\begin{array}{l}\text { Telescope / } \\
\text { Instrument }\end{array}$ & $\begin{array}{c}\text { Grating / } \\
\text { Central Wavelength }(\AA)\end{array}$ & $\begin{array}{l}\text { Exposure } \\
\text { (s) }\end{array}$ & Observer ${ }^{\mathrm{b}}$ \\
\hline \multicolumn{6}{|c|}{ UGC 2773 OT2009-1 } \\
\hline 15.1 & 2009 Sep. 2.6 & Gemini/GMOS & $\mathrm{R} 400 / 7000$ & $2 \times 1200$ & $\mathrm{KO}, \mathrm{RM}$ \\
\hline 32.9 & 2009 Sep. 20.4 & MMT/Blue Channel & $300 / 5787$ & $3 \times 1200$ & $\mathrm{PC}$ \\
\hline 33.9 & 2009 Sep. 21.4 & MMT/Blue Channel & $300 / 5787$ & 1200 & $\mathrm{PC}$ \\
\hline 33.9 & 2009 Sep. 21.4 & MMT/Blue Channel & $832 / 4029$ & $2 \times 900$ & $\mathrm{PC}$ \\
\hline 34.0 & 2009 Sep. 21.5 & MMT/Blue Channel & $832 / 4830$ & $2 \times 900$ & $\mathrm{PC}$ \\
\hline 34.0 & 2009 Sep. 21.5 & MMT/Blue Channel & $832 / 6563$ & $3 \times 900$ & $\mathrm{PC}$ \\
\hline 95.8 & 2009 Nov. 22.3 & MMT/Blue Channel & $300 / 5787$ & $2 \times 1800$ & $\mathrm{PC}$ \\
\hline \multicolumn{6}{|c|}{ SN 2009ip } \\
\hline 3.5 & 2009 Sep. 1.3 & Clay/MagE & & $3 \times 900$ & II \\
\hline 22.5 & 2009 Sep. 20.3 & MMT/Blue Channel & $300 / 5787$ & $4 \times 1200$ & $\mathrm{PC}$ \\
\hline 23.5 & 2009 Sep. 21.2 & MMT/Blue Channel & $832 / 4029$ & $2 \times 1200$ & PC \\
\hline 23.5 & 2009 Sep. 21.3 & MMT/Blue Channel & $832 / 4830$ & $2 \times 1200$ & $\mathrm{PC}$ \\
\hline 23.5 & 2009 Sep. 21.3 & MMT/Blue Channel & $832 / 6563$ & 1200 & $\mathrm{PC}$ \\
\hline 85.6 & 2009 Nov. 22.1 & MMT/Blue Channel & $300 / 5787$ & $3 \times 1200$ & $\mathrm{PC}$ \\
\hline
\end{tabular}

${ }^{a}$ Days since maximum, MJD 55,061.5 and 55,071.8 for UGC 2773 OT2009-1 and SN 2009ip (S09), respectively.

${ }^{\mathrm{b}} \mathrm{II}=\mathrm{I}$. Ivans, $\mathrm{KO}=\mathrm{K}$. Olsen, $\mathrm{PC}=\mathrm{P}$. Challis, $\mathrm{RM}=\mathrm{R}$. McDermid

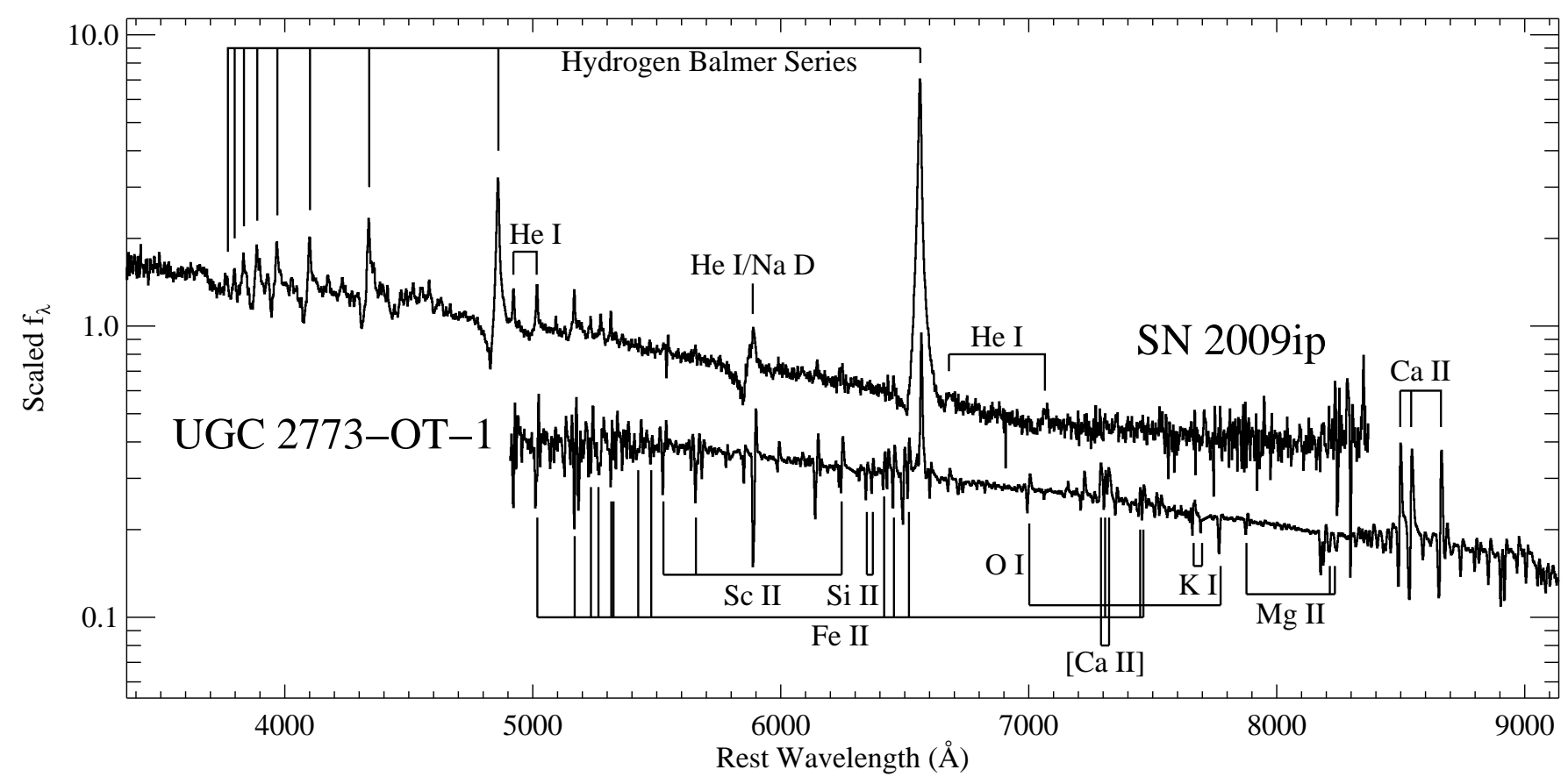

Figure 2. Optical spectra of SN 2009ip and UGC 2773 OT2009-1. The spectrum of UGC 2773 OT2009-1 has been dereddened by $E(B-V)=0.564 \mathrm{mag}$. Several lines are identified and marked.

We have also performed this procedure on several stars in the vicinity of the progenitor of UGC 2773 OT20091. Assuming that all of these stars are part of a cluster and were formed at the same time, they should place additional limits on the current maximum-mass stars of the cluster. These stars are all consistent with an initial mass of $M_{\odot} \lesssim 25 M_{\odot}$. There is a single star that is particularly blue (and therefore potentially very massive), but it is still consistent with an initial mass of $25 M_{\odot}$. The likely association of the progenitor of UGC 2773
OT2009-1 with this cluster and its upper mass limit of $\sim 25 M_{\odot}$ further supports the initial mass estimate for the progenitor of UGC 2773 OT2009-1.

Considering the blue colors of the stars in the cluster, it is unlikely that they are significantly reddened by host galaxy dust. As shown in Figure 3. a relatively small amount of extinction could significantly increase our initial mass estimate for UGC 2773 OT2009-1. In Section 3.4 we show that there was likely a significant amount of circumstellar dust existing before the out- 


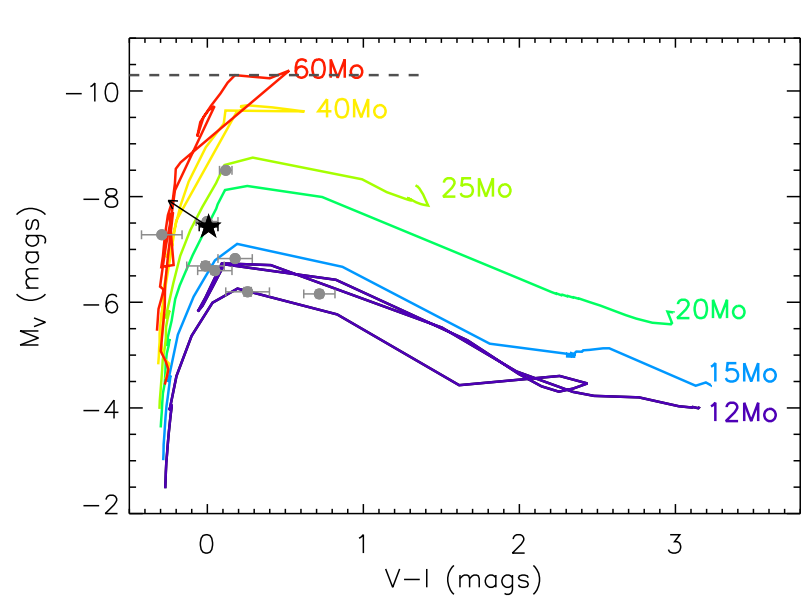

Figure 3. Color-magnitude diagram $\left(V-I\right.$ vs. $\left.M_{V}\right)$ for the progenitor of UGC 2773 OT2009-1 (star) and stars spatially located within the same star cluster (grey circles). The measurements have been corrected for the Milky Way extinction of $A_{V}=1.75 \mathrm{mag}$ and $E(V-I)=0.902 \mathrm{mag}$, but no host or circumstellar extinction is assumed for the stars. For comparison, solar metallicity, nonrotating, "standard" mass loss stellar evolution tracks are also plotted (Schaller et al. 1992). The progenitor of UGC 2773 OT2009-1 has the same colors and absolute magnitude of a $20 M_{\odot}$ model. The stars in the cluster are consistent with the models of stars with ZAMS masses $\leq 25 M_{\odot}$, but a single star is also consistent with a much higher mass. The arrow represents $A_{V}=0.5 \mathrm{mag}$ of additional extinction (assuming $R_{V}=3.1$ ). The dashed line represents the absolute magnitude of the progenitor of SN 2009ip with a reasonable range of colors. The luminosity of the progenitor of SN 2009ip is consistent with an initial mass of $\gtrsim 60 M_{\odot}$.

burst, indicating that the progenitor had an initial mass much larger than the reddening-free estimate of $20 M_{\odot}$.

The combination of the reddening-free initial mass estimate for the progenitor of UGC 2773 OT2009-1, the initial mass estimates of stars likely within the same cluster as the progenitor, and the probably circumstellar dust extinction give us a conservative lower limit on the initial mass of the progenitor of UGC 2773 OT2009-1 of $\sim 25 M_{\odot}$.

\subsection{Spectroscopic Comparisons}

\subsubsection{SN 2009ip}

We present the 24 and 86 day spectra of SN 2009ip in Figure 4. In the upper panel of Figure 4 the 24 day spectrum is compared to the 2 day spectrum of the LBV outburst SN 1997bs (Van Dyk et al. 2000). Both objects have blue continua, strong and narrow $\mathrm{H}$ Balmer lines, and $\mathrm{He}$ I and Fe II emission lines. Unlike SN 1997bs, SN 2009ip has a particularly strong He I $\lambda 5876$ line (with some possible contribution from $\mathrm{Na} \mathrm{D}$ ), and all $\mathrm{H}$ Balmer lines and He I $\lambda 5876$ show strong absorption features with minima blueshifted by $\sim 3000 \mathrm{~km} \mathrm{~s}^{-1}$ (see Section 3.3.1 for a detailed discussion of this high-velocity absorption).

Inspecting the 25 day spectrum from $\mathbf{S 0 9}$, we see some indication of the $3000 \mathrm{~km} \mathrm{~s}^{-1}$ absorption component, particularly for $\mathrm{H} \beta$ and $\mathrm{He} \mathrm{I} \lambda 5876$; however, the absorption is much stronger in the spectra presented here (which were obtained at very similar times). We have reduced our spectra with many different extraction regions and backgrounds, with the high-velocity absorption fea-

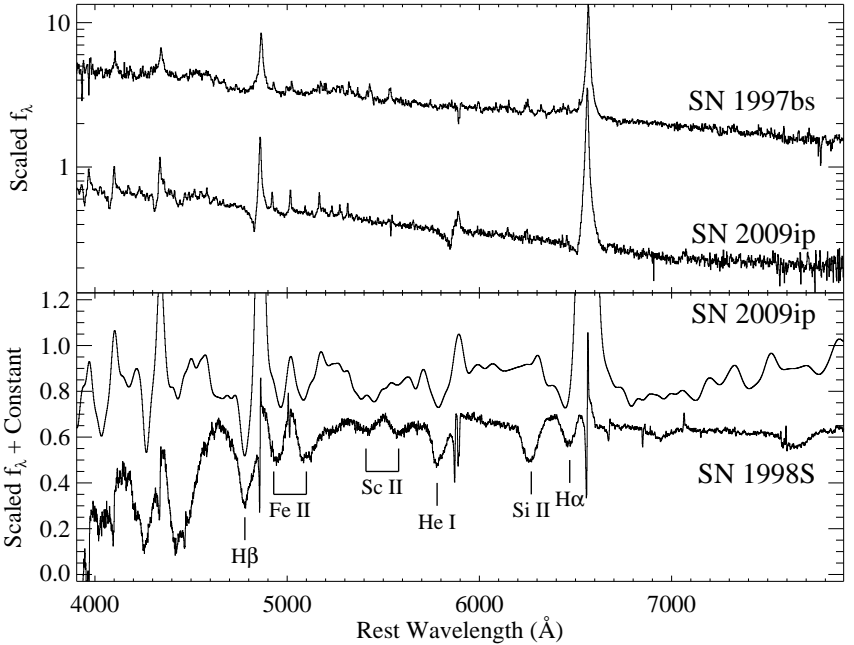

Figure 4. Top panel: the 23 day optical spectrum of SN 2009ip compared to the 2 day spectrum of SN 1997bs (Van Dyk et al. 2000). Bottom panel: the 86 day optical spectrum of SN 2009ip after smoothing and subtracting a 10,000 K blackbody (see text for details). For comparison, the 25 day spectrum of SN 1998S (after subtracting a 10,000 K blackbody) is also shown (Leonard et al. 2000). Prominent, high-velocity lines have been marked.

tures are present in all reductions. The same feature is present in all spectra taken with the MMT on days 23 and 24 , which were taken with different gratings and wavelength regions. It is also present on day 86 , but with a different velocity. The absorption is present for all Balmer lines and He I $\lambda 5876$. Despite the apparent differences with the concurrent Keck spectrum (S09), we are confident that the high-velocity absorption features are real and not an artifact of the spectral reductions.

The bottom panel of Figure 4 displays the spectra of SN 2009ip and SN IIn 1998S (after subtracting a $10,000 \mathrm{~K}$ blackbody spectrum from both) from 86 and 25 days, respectively. An inverse-variance weighted Gaussian filter (with a width of $1000 \mathrm{~km} \mathrm{~s}^{-1}$ ) has been applied to the spectrum of SN 2009ip (Blondin et al. 2006). This filtering will smear out features with intrinsic widths less than $1000 \mathrm{~km} \mathrm{~s}^{-1}$, but will appropriately smooth features on larger scales. The high-velocity absorption in the 86 day spectrum of SN 2009ip is at a higher velocity than at 24 days. At this epoch, the velocity of the fast-moving SN 2009ip ejecta are very similar to that of SN 1998S. Although the H Balmer emission lines are much stronger in SN 2009ip, most other features are similar in the two spectra. In particular, SN 2009ip shows the H Balmer, He I, Sc II, and Fe II features seen in SN 1998S. SN 2009ip is missing the strong absorption at $6250 \AA$ that is attributed to Si II in SN $1998 \mathrm{~S}$ (Leonard et al. 2000). This feature may be the result of a significant amount of nuclear burning, and thus not present in the ejecta of SN 2009ip.

\subsubsection{UGC 2773 OT2009-1}

As discussed in Section 2.5, UGC 2773 OT2009-1 has a spectrum with narrow $\mathrm{H} \alpha$ emission, [Ca II] emission, and P-Cygni absorption from many intermediate-mass and Fe-group elements. Perhaps the most distinguishing feature compared to other massive star outbursts is the [Ca II] emission. In Figure 5, we compare the 15 day 


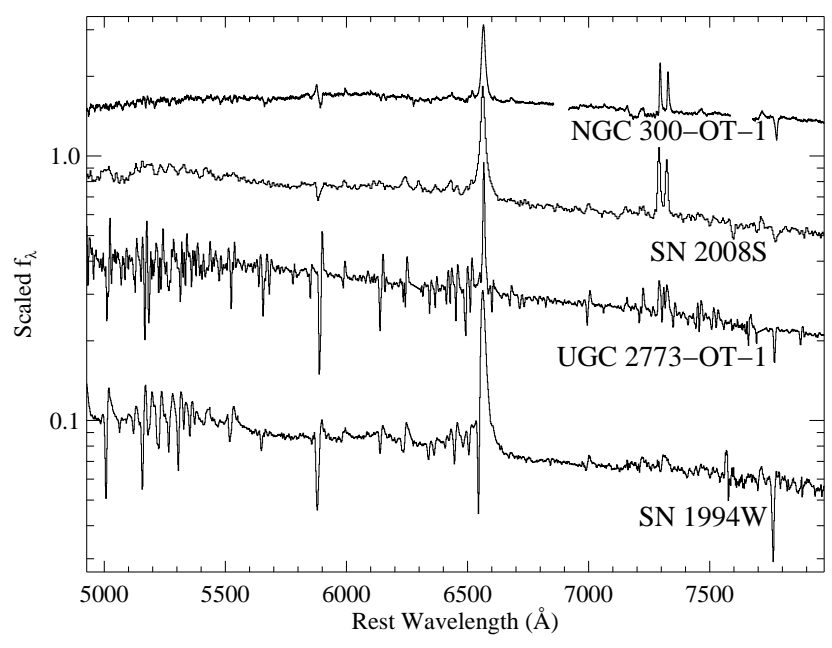

Figure 5. Optical spectra of UGC 2773 OT2009-1, NGC 300 OT2008-1 (Berger et al. 2009b), SN 1994W (Chugai et al. 2004), and SN 2008S (Smith et al. 2008b). All spectra have narrow $\mathrm{H} \alpha$ and [Ca II] emission; however, NGC 300 OT2008-1 and SN 2008S lack the forest of lines (especially Fe II) that UGC 2773 OT2009-1 and SN 1994W display.

spectrum of UGC 2773 OT2009-1 to spectra of the lowluminosity transients NGC 300 OT2008-1 (Berger et al. 2009b) and SN 2008S (Smith et al. 2008b), as well as SN IIn 1994W (Chugai et al. 2004); all of these objects have [Ca II] emission in their spectra.

All spectra in Figure 5 are relatively similar. The continuum of each spectrum is well-described by a blackbody spectrum, with all four objects having a similar temperature. Each object has a prominent $\mathrm{H} \alpha$ emission line, with UGC 2773 OT2009-1 having a narrower line than the other objects. Additionally, SN 1994 W has a strong $\mathrm{H} \alpha$ absorption line blueward of its emission peak.

NGC 300 OT2008-1 and SN 2008S are very similar objects with massive $\left(10-25 M_{\odot}\right)$, dusty progenitors (Prieto et al. 2008; Berger et al. 2009b; Bond et al. 2009). Their spectra share many characteristics with the yellow hypergiant IRC+10240 (Smith et al. 2008b). Although UGC 2773 OT2009-1 shares some spectroscopic properties with these two transients and IRC+10240 (see S09 for additional discussion), the latter objects lack the forest of absorption lines in UGC 2773 OT2009-1. These lines are reminiscent of an F-type supergiant. The PCygni profiles of these lines and the hydrogen Balmer emission are very similar to S Dor during a cool phase (e.g., Massey 2000).

SN $1994 \mathrm{~W}$ was very luminous at peak $\left(M_{V} \approx\right.$ -19 mag), but generated at most $0.03 \quad M_{\odot}$ of ${ }^{56} \mathrm{Ni}$ (Sollerman. Cumming. \& Lundqvist 1998). Dessart et al. (2009) presented an alternative method of producing the photometric and spectroscopic properties of this object: the collision of two massive hydrogen shells ejected from the star with no core collapse. Spectra of SNe IIn are rather heterogeneous (see Figure 5 of Smith et al. 2009a for a comparison of various objects), and SN $1994 \mathrm{~W}$ is relatively distinct for its narrow absorption features. Given the spectral similarity between UGC 2773 OT2009-1 and SN 1994W, the strict upper limit of ${ }^{56} \mathrm{Ni}$ mass in SN $1994 \mathrm{~W}$, and the alternative model of Dessart et al. (2009), one must further question if SN 1994W destroyed its progenitor star.

\subsubsection{Contrasting SN 2009ip and UGC 2773 OT2009-1}

At $t=0$ days, the temperature of UGC 2773 OT2009-1 is $\sim 7000 \mathrm{~K}$ (see Section [3.4), which is similar to the temperature during the "eruptive" state of LBVs (e.g., Humphreys \& Davidson 1994). This contrasts with the higher temperature of $10,000 \mathrm{~K}$ derived for SN 2009ip (see Section 3.4), which lacks the narrow Fe-group absorption features. Many other LBV giant eruptions have temperatures similar to that of SN 2009ip (Humphrevs \& Davidson 1994). SN 2009ip was $~ 2$ mag brighter at peak than UGC 2773 OT2009-1, and SN 2009ip had a much larger increase in luminosity during the year before maximum than UGC 2773 OT2009-1, increasing by $\gtrsim 5$ mag and $\sim 1$ mag over one year, respectively (S09). The fast-moving ejecta of SN 2009ip also contrasts with the relatively slow outflow of UGC 2773 OT2009-1.

The photometric and spectroscopic differences of these objects suggests different physical mechanisms. Clearly a supersonic explosion is necessary to produce the high-velocity absorption features of SN 2009ip, while UGC 2773 OT2009-1 shows no indication of an explosion. The differences in temperature and luminosity increase are also indicative of more energy injection (per unit mass) for SN 2009ip. A plausible explanation is that SN 2009ip is a LBV giant eruption triggered by an explosion, while UGC 2773 OT2009-1 is a particularly luminous S Dor eruption.

\subsection{Line Profiles}

In this section, we examine the line profiles of $\mathrm{H} \alpha$ and Ca lines. These features provide an indication of the kinematics of the emitting material. The narrow lines are a tracer of the pre-shock circumstellar material, while the high-velocity absorption features in the spectra of SN 2009ip probe the outburst ejecta.

\subsection{1. $H \alpha$}

In Figure 6. we present the $\mathrm{H} \alpha$ line profiles of UGC 2773 OT2009-1 and SN 2009ip. Three separate epochs are shown for each object. Both objects have asymmetric line profiles. There are absorption components at about $-350 \mathrm{~km} \mathrm{~s}^{-1}$ for UGC 2773 OT2009-1 and between -3000 and $-6000 \mathrm{~km} \mathrm{~s}^{-1}$ for SN 2009ip. The line profile of SN 2009ip has a different shape and is much broader than that of UGC 2773 OT2009-1. We have attempted to fit these line profiles, but because of the asymmetry of the profiles, we first fit only the redshifted portion of each line profile and then add an absorption component to reproduce the blueshifted profile.

For the 15 day spectrum of UGC 2773 OT2009-1, we fit a Gaussian with FWHM $=780 \mathrm{~km} \mathrm{~s}^{-1}$ to the red side of the feature. This value is twice that of the value found by $\mathbf{S 0 9}$ for a spectrum from day 22, but examination of their figures suggest that they reported half-width at half maximum (HWHM) or the standard deviation of the Gaussian fit (which is smaller by a factor of 2.35) rather than FWHM. The 34 day spectrum of UGC 2773 OT2009-1 is contaminated by host-galaxy emission lines, 


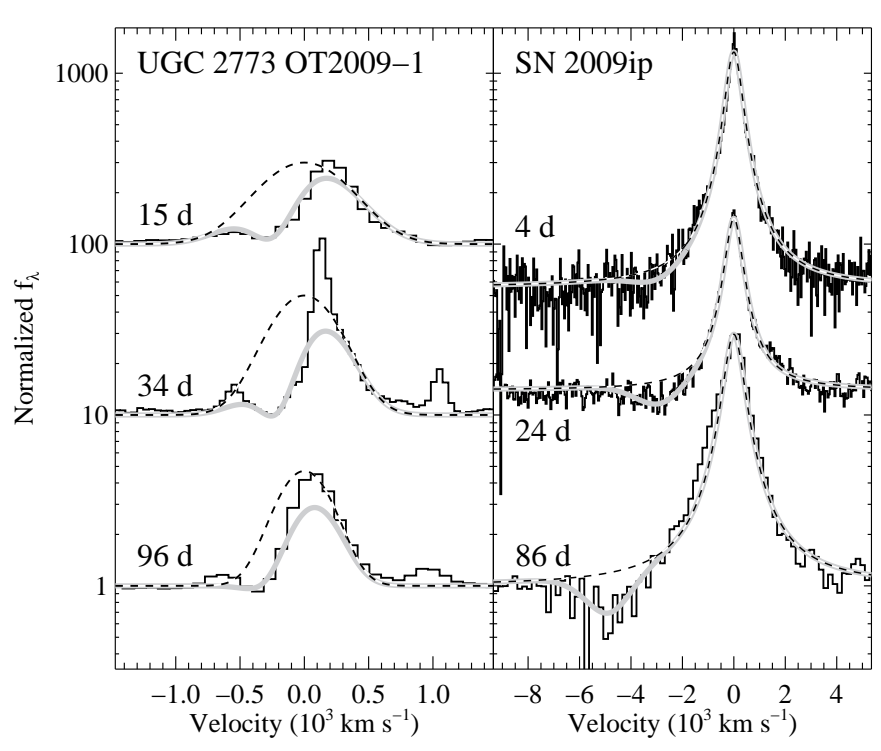

Figure 6. Normalized spectra of UGC 2773 OT2009-1 and SN 2009ip near $\mathrm{H} \alpha$. The line profiles are fit with Gaussian and Lorentzian profiles, respectively. A profile fit to the redshifted portion of each profile is shown as a dashed line. The grey lines correspond to the redshifted profile with a Gaussian absorption component added. Narrow [N II] can be seen in the spectrum of UGC 2773 OT2009-1.

making a fit to the inner regions of the line profile problematic. Ignoring this region, we were able to fit the redshifted portion of the line profile with a single Gaussian with FWHM $=590 \mathrm{~km} \mathrm{~s}^{-1}$. The 96 day spectrum has lower resolution, but is successfully fit by a Gaussian profile with $\mathrm{FWHM}=470 \mathrm{~km} \mathrm{~s}^{-1}$.

To account for the asymmetric profile, we add an absorption component to the Gaussian line profiles. Fitting the full profile with two Gaussian functions, the emission component fit to the red side of the line and the absorption component added to fit the blue side of the line, we find absorption minima at $-180,-110$, and $-80 \mathrm{~km} \mathrm{~s}^{-1}$ for the 15,34 , and 96 day spectra, respectively. This is different from the value of the actual minimum $\left(-350 \mathrm{~km} \mathrm{~s}^{-1}\right)$ since the relatively strong emission masks the true minimum.

The line profiles of the first two spectra (days 4 and 24) of SN 2009ip are well fit by Lorentzian profiles with FWHM $=780 \mathrm{kms}^{-1}$ and the third is best fit by a Lorentzian profile with FWHM $=890 \mathrm{kms}^{-1}$, which are larger than that found by $\mathrm{S} 09,550 \mathrm{~km} \mathrm{~s}^{-1}$. A Lorentzian profile of $550 \mathrm{~km} \mathrm{~s}^{-1}$ is not a particularly bad fit to our data, but we find that the larger velocities better represent the data. One can also see in Figure 8 of S09, that the $550 \mathrm{~km} \mathrm{~s}^{-1}$ Lorentzian slightly underpredicts the true FWHM of the line, so the data appear to be consistent.

In the 24 day spectrum of SN 2009ip, we see an absorption feature with a minimum at a velocity of about $-3000 \mathrm{kms}^{-1}$. (This high-velocity absorption is seen for all Balmer lines with varying instrument configurations and on two epochs; see Section 3.2.1.) This feature is well fit by including a Gaussian absorption component with a minimum at $-2800 \mathrm{~km} \mathrm{~s}^{-1}$. Adding a component with this velocity also improves the fit to the 4 day $\mathrm{H} \alpha$ profile slightly, but not in a significant way. The 86 day spectrum shows an even stronger high-velocity absorption component with the minimum of the absorption at a larger velocity of $-4800 \mathrm{~km} \mathrm{~s}^{-1}$. The blue wing of the absorption component, representing the fastest moving material, corresponds to a velocity of about -4500 and $-7000 \mathrm{~km} \mathrm{~s}^{-1}$ for the 24 and 86 day spectra, respectively.

These velocities are significantly larger than the outflow velocity of $550 \mathrm{~km} \mathrm{~s}^{-1}$ assumed by S09. They are much larger than the wind speed of LBVs and are larger than the measured velocity for any LBV eruption with the exception of the 1843 eruption of $\eta$ Car, which had some material expelled at $3000-6000 \mathrm{~km} \mathrm{~s}^{-1}$ (Smith 2008). The velocities measured for SN 2009ip are similar to that of the ejecta of typical core-collapse SNe (such as SN 1998S; see Figure 4 and Section 3.2.1) and are somewhat similar to that of Wolf-Rayet winds (e.g., Abbott \& Conti 1987). We discuss the implications of these features in Section 4.2 .

\subsubsection{Permitted and Forbidden Ca II}

Only our first spectrum of SN 2009ip covers the Ca II NIR triplet, and no spectrum shows obvious [Ca $\mathrm{CI}]$ $\lambda \lambda 7291,7325$ lines, similar to the spectra presented by S09. Furthermore, the $\mathrm{Ca} \mathrm{H} \& \mathrm{~K}$ lines are confused by the strong Balmer sequence in SN 2009ip. Because of these factors, it is difficult to evaluate the characteristics of the Ca II behavior in this object (other than the absent [Ca II] lines).

UGC 2773 OT2009-1, on the other hand, has strong Ca II features. This can be seen in Figure 2. We examine the $\mathrm{Ca} \mathrm{H} \& \mathrm{~K}$, [Ca II] $\lambda \lambda 7291,7325$, and Ca II NIR triplet line profiles in Figure 7 . The $\mathrm{Ca} \mathrm{H} \& \mathrm{~K}$ lines show a broad absorption extending from -1000 to $+500 \mathrm{~km} \mathrm{~s}^{-1}$ and a minimum at about $-50 \mathrm{~km} \mathrm{~s}^{-1}$ that does not appear to change significantly between the two epochs. Each component of the Ca II NIR triplet shows a strong P-Cygni profile with a minimum at approximately $-250 \mathrm{~km} \mathrm{~s}^{-1}$, slightly larger than the minima of Ca H\&K.

The [Ca II $] \lambda \lambda 7291,7325$ lines are visible in all epochs of our spectroscopy. We confirm the additional line between this doublet seen by S09 and identify this as Fe II $\lambda 7308$. Both [Ca II] lines have asymmetric profiles in all spectra; the peak is at zero velocity, but the emission extends further to the red than to the blue. The lines from all epochs have FWHMs of $\sim 400 \mathrm{~km} \mathrm{~s}^{-1}$, which is about half the width of $\mathrm{H} \alpha$ (see Section 3.3.1), similar to that found for NGC 300 OT2008-1 (Berger et al. 2009b).

\subsection{Spectral Energy Distribution and Dust Emission}

Using our available photometry and spectroscopy, we can examine the spectral energy distribution (SED) of both objects. We have only optical spectra of SN 2009ip, which limits our ability to examine multiple blackbody components for this object. A 10,000 K blackbody fits our optical spectra well, which is consistent with that found by $\mathrm{S} 09$.

Our single epoch of Swift photometry occurred during the dramatic fading of the light curve immediately following maximum brightness (S09). In Figure 8 , the Swift photometry is combined with the unfiltered photometry (approximately $R$ band) of $\mathrm{S} 09$ (with an uncertainty of 0.5 mag to account for the 16 hour difference in the epoch of the observations) during the minimum. We overplot 


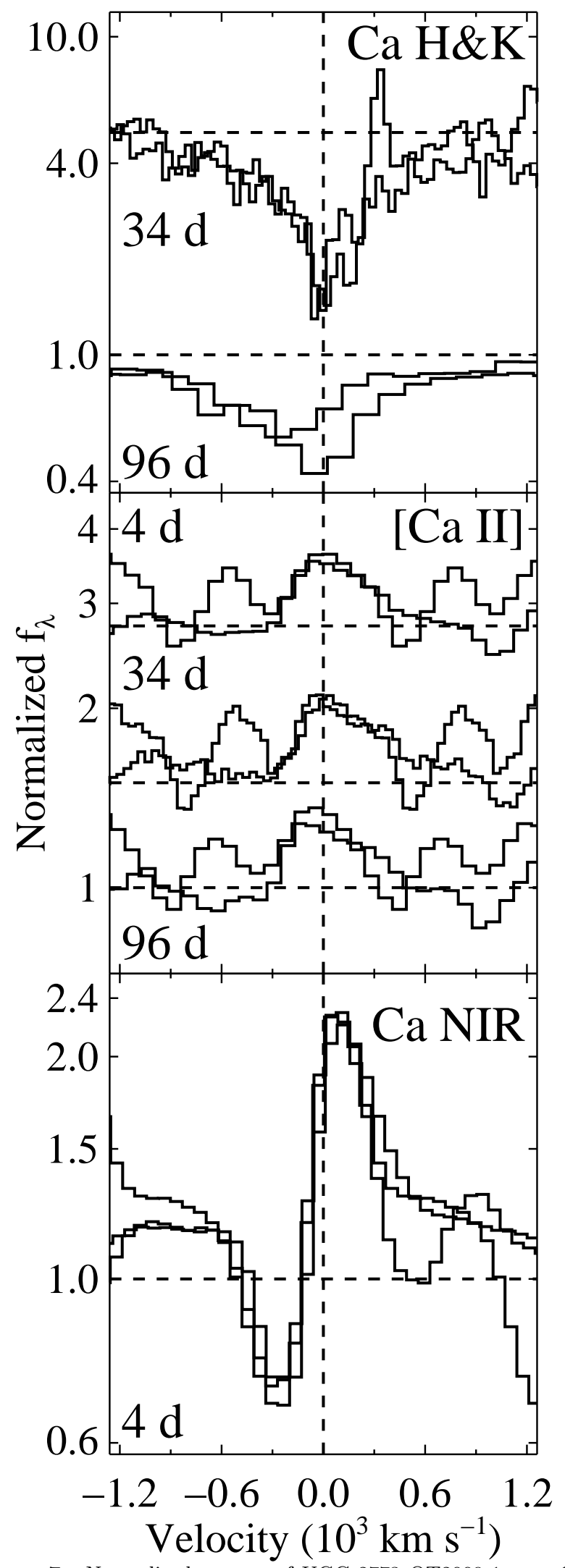

Figure 7. Normalized spectra of UGC 2773 OT2009-1 near Ca H\&K (top), [Ca II] $\lambda \lambda 7291,7325$ (middle), and Ca II NIR triplet (bottom). Dashed lines indicate the continuum flux and zero velocity for each line. Each member of the multiplet is overplotted for a given spectrum.

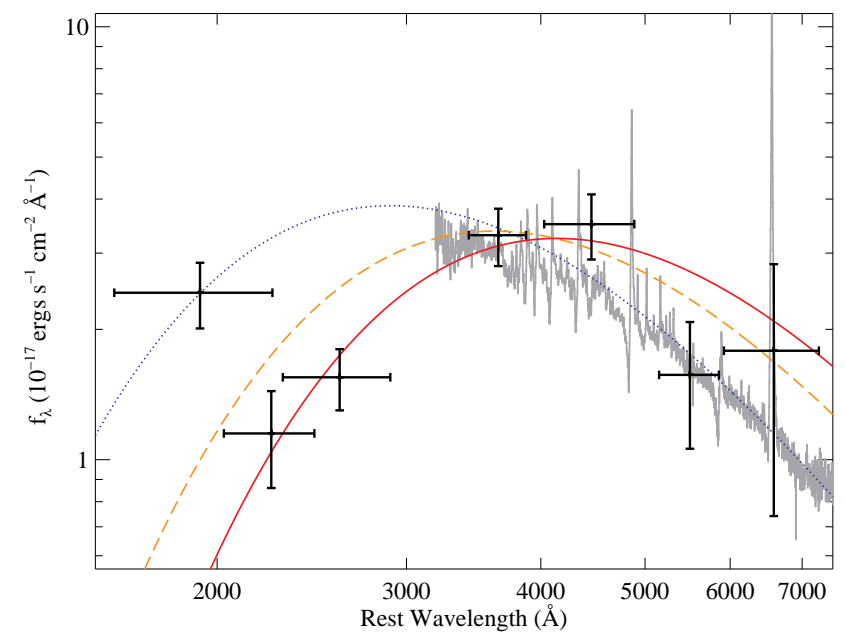

Figure 8. UV/Optical photometry of SN 2009ip during the fading event immediately after maximum brightness. The blue dotted, orange dashed, and red solid curves correspond to $10,000,8000$, and $7000 \mathrm{~K}$ blackbody spectra, respectively. The 23 day spectrum is also plotted to show the consistency with both the photometry and the 10,000 K blackbody. All photometry is consistent with the 8000 and 10,000 K blackbody spectra. Ignoring the bluest (UVW2) filter, the data are also consistent with the $7000 \mathrm{~K}$ blackbody.

the 23 day spectrum for comparison. The optical photometry is consistent with the optical spectrum and a $10,000 \mathrm{~K}$ blackbody. The UVW2 flux is also consistent with this blackbody, however, the UVM2 and UVW1 measurements fall well below this curve. Although this may be the result of line blanketing, these data are also consistent with a blackbody curve with a temperature as low as $8000 \mathrm{~K}$. If we ignore the UVW2 measurement, the data can be fit by a $7000 \mathrm{~K}$ blackbody. Although our data suggest a possible change in the SED during the fading event, the lack of necessary comparison UV data from a different epoch prevent a clear indication of a change.

Using the 15 day optical spectrum and 22 day NIR spectrum, we are able to examine the SED of UGC 2773 OT2009-1 over nearly a decade in wavelength. Between these dates, the light curve of UGC 2773 OT2009-1 was essentially constant, having the same magnitude (within $1 \sigma)(\mathrm{S} 09)$. Using the long wavelengths of the NIR spectrum, our data are sensitive to any low-temperature thermal components.

We fit a single blackbody to these data, ignoring regions with strong line features and simultaneously fitting the scaling between the optical and NIR spectra. Doing this results in a best-fit temperature of $6800 \mathrm{~K}$. This single blackbody consistently under-predicts the flux at NIR wavelengths. As a result, we have also attempted to fix the spectrum with a double blackbody model. This model, which produces a much better fit, results with temperatures $T_{1}=6950 \mathrm{~K}$ and $T_{2}=2100 \mathrm{~K}$. The full spectrum and associated fits are shown in Figure 9 .

S09 noted that UGC 2773 OT2009-1 had a (photometric) NIR excess, but could not distinguish between circumstellar extinction and dust emission. To test the former case, we attempted to fit the spectrum with a single blackbody, but with an additional extinction term. With $R_{V}$ fixed to 3.1 , this model did not fit the data 


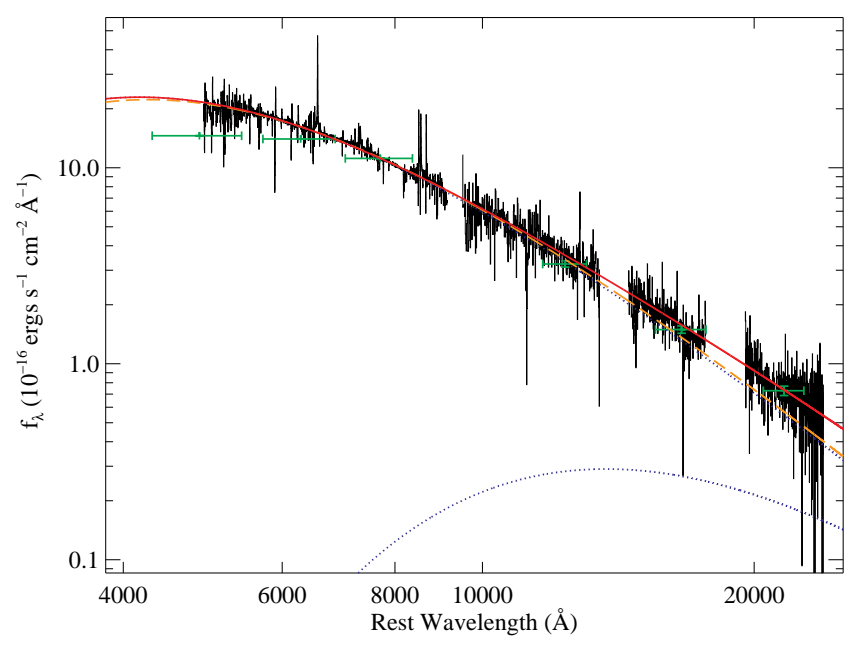

Figure 9. Optical/NIR spectrum of UGC 2773 OT2009-1. Single (6800 K; dashed orange line) and double (2100 and $6900 \mathrm{~K}$; solid red line) blackbody fits to the spectrum are overplotted. The individual components of the double blackbody fit are shown as blue dotted lines. The double blackbody is a better fit to the data than the single blackbody. Green points show our photometry, which also shows a NIR excess. The $g$-band flux is below that of either curve, but that is likely the result of line blanketing.

well. The model was able to sufficiently reproduce the data if we allowed $R_{V}<1$, which is unphysical. We therefore conclude that the NIR excess is likely due to dust emission.

Scaling our spectrum to our broad-band photometry, we can calibrate the blackbody flux, which in turn constrains the ratio $R / D$, where $R$ is the radius of the blackbody radiation and $D$ is the distance to the object. Using $D=6 \pm 0.5 \mathrm{Mpc}$, we find that the hot and cool blackbodies have radii of $(1.50 \pm 0.16) \times 10^{14} \mathrm{~cm}$ and $(4.3 \pm 0.4) \times 10^{14} \mathrm{~cm}(13.0 \pm 1.1 \mathrm{AU}$ and $29 \pm 2 \mathrm{AU})$, respectively. The size of the cool emitting region is of the same order of magnitude of the size of the Homunculus nebula surrounding $\eta$ Car.

Following the prescription outlined by Smith, Foley, \& Filippenko (2008a) (and references therein), we can measure the mass of the emitting dust. Specifically,

$$
M_{d}=\frac{400 \pi \rho R_{d}^{2}}{3 T_{d}^{2}},
$$

where $M_{d}$ is the dust mass, $R_{d}$ is the radius of the dust, $T_{d}$ is the dust temperature, and $\rho$ is the dust density. For the values obtained from the spectra, $T_{d}=2100 \mathrm{~K}$ and $R_{d}=4.3 \times 10^{14} \mathrm{~cm}$, and assuming a dust grain density of $\rho=2.25 \mathrm{~g} \mathrm{~cm}^{-3}$, we find a dust mass of $M_{d} \approx$ $2 \times 10^{-8} M_{\odot}$. Since there could be a significant amount of dust emission at lower temperatures, this is a lower limit on the total dust mass; however, it is worth noting that this measurement is orders of magnitude less than the dust created in some SNe (e.g., Kotak et al. 2009, and references therein). We note depending on the dust composition, the dust temperature may differ from the blackbody temperature by hundreds of degrees.

The dust is very close to the star and its temperature is near the limit of grain survival. Given these conditions, it is very likely that pre-existing circumstellar dust was heated and is emitting as it is being vaporized, rather than newly formed dust emitting as it cools.

\section{DISCUSSION}

\subsection{Different Massive Star Outbursts}

UGC 2773 OT2009-1 and SN 2009ip provide excellent examples of the diversity of massive star outbursts. UGC 2773 OT2009-1 increased its optical brightness by $\sim 1$ mag during outburst and has a cool spectrum with many narrow absorption lines and [Ca II] emission. It occurred near a star cluster containing stars with initial masses of $\sim 25 M_{\odot}$ and shows evidence for a very cool $(T \approx 2100 \mathrm{~K})$ thermal component that is radiated by circumstellar dust. SN 2009ip had a more massive and relatively isolated progenitor. At peak, it had risen at least 4 mag over the previous year, and its spectrum was hot and dominated by $\mathrm{H}$ Balmer emission lines. After having a significant fading and rebrightening over three weeks, it developed high-velocity absorption lines. Its SED is consistent with no dust emission.

The high temperature and large increase in optical luminosity for SN 2009ip indicates that it was a true giant eruption akin to the 1843 eruption of $\eta$ Car. UGC 2773 OT2009-1, on the other hand, has spectral characteristics similar to that of $\mathrm{S}$ Dor at maximum. The relatively small increase in optical luminosity may indicate that UGC 2773 OT2009-1 was the result of normal S Dor variability, but that it was a particularly luminous maximum. While the largest normal variation of S Dor stars vary by $\sim 3$ mag in the optical (van Genderen 2001), UGC 2773 OT2009-1 has varied by at least $\sim 5 \mathrm{mag}$ (including the current eruption) over the last ten years (Section 2, see also S09).

The outbursts of NGC 300 OT2008-1 and SN 2008S both had relatively low temperature SEDs (Berger et al. $2009 \mathrm{~b})$, but neither had the forest of absorption lines found in UGC 2773 OT2009-1. All three objects had [Ca II] emission, but as suggested by [S09, this may be linked to the circumstellar environment, and particularly dust destruction, rather than the event. Our observations have shown that there is dust in the circumstellar environment of the progenitor, and that it was likely pre-existing dust that is in the process of being vaporized. Additionally, SN 1999bw had [Ca II] emission (Garnavich et al. 1999) and had an IR excess consistent with dust emission at late times (Sugerman et al. 2004). All four massive star outbursts with observed [Ca II] emission (SN 1999bw, SN 2008S, NGC 300 OT2008-1, and UGC 2773 OT2009-1) have evidence of circumstellar dust. We do note that SN 2000ch had a $\sim 7000 \mathrm{~K}$ spectrum and an infrared excess consistent with dust emission, but no strong [Ca II] emission was detected in the relatively low signal-to-noise spectra presented by Wagner et al. (2004). It is therefore possible to have a cool object and circumstellar dust yet not have [Ca II] emission.

Although UGC 2773 OT2009-1, NGC 300 OT2008-1, and SN 2008S have circumstellar dust and similar temperatures, other than the narrow $\mathrm{H}$ Balmer and [Ca II] (which is linked to the presence of circumstellar dust) emission, the spectra and progenitors are not particularly similar. Particularly, NGC 300 OT2008-1 and SN 2008S had relatively featureless spectra and progenitors with 
initial masses of $10-25 M_{\odot}$, while UGC 2773 OT20091 had spectrum dominated by narrow lines and a more massive progenitor $\left(\gtrsim 25 M_{\odot}\right)$. Additional data are necessary to determine if the outburst mechanisms in these objects are similar.

Using SN 2009ip and UGC 2773 OT2009-1 as examples, there appears to be two distinct elements that determine the observational properties of massive star outbursts. The first is the temperature of the outburst, which may be related to the increase in luminosity, the instability that causes the eruption, the width of the emission lines, and possibly the energetics of the outburst and if there is an explosion (see Section 4.2). This directly determines the shape of the optical SED, the ionization, if there is a forest of absorption lines, and possibly the shape of the line profiles, if there is high-velocity absorption. The other characteristic is the amount of circumstellar dust, which may cause strong Ca emission (and particularly [Ca II] emission) and will determine the shape of the SED at longer wavelengths. UGC 2773 OT2009-1 and SN 2009ip would occupy very different regions of the parameter space created by these two dimensions. NGC 300 OT2008-1 and SN 2008S would be close to UGC 2773 OT2009-1, while LBV giant eruptions such as SN 1997bs would be close to SN 2009ip.

It remains to be seen if there are hot massive star outbursts with a large amount of circumstellar dust or if there are cool massive star outbursts with little circumstellar dust. $\eta$ Car has $0.125 M_{\odot}$ of dust surrounding it (Smith et al. 2003); if it were to have another giant eruption today, would it be cool? SN 1999bw had [Ca II] emission and displayed dust emission at late times; was it hot? An IR survey of recent massive star outbursts with good spectroscopic coverage may provide these answers. In the future, optical and NIR observations may be sufficient to determine these characteristics for other massive star outbursts.

\subsection{SN 2009ip: A Supersonic Explosion}

The spectra of SN 2009ip have absorption attributed to high-velocity (up to $\sim 7000 \mathrm{~km} \mathrm{~s}^{-1}$ ) material (see Section 3.3.1). Contrary to what is expected from a single outburst or explosion, the velocity of the absorption feature increases with time. In a typical SN, the ejecta naturally follow a Hubble law with the highest velocity material being the most distant from the explosion site. Spectral lines have a blueshifted absorption due to the scattering processes in the photosphere of the SN. Low velocity material is hidden behind the photosphere, only to be revealed at later times. As the photosphere recedes, the highest-velocity material becomes optically thin, resulting in the blueshifted velocity of a spectral line to decrease with time. Since the absorbing material must be at just slightly larger radii than the photosphere, the high-velocity material must have been ejected during the eruption. (If the absorbing material were from a previous eruption, the ejecta from the more recent eruption would have had to be moving even faster.)

It is possible that the high-velocity absorption is a component of P-Cygni features from the ejected material. The Lorentzian profile slightly underestimates the emission flux in the 86 day spectrum (see Figure 6), which may be the result of P-Cygni emission contributing to the line. Since the high-velocity absorption is coming from the ejecta, the outburst of SN 2009ip must have been extremely energetic, expelling a large amount of material at very high velocities. However, for the velocity to increase with time, either the ejecta must not follow a Hubble expansion or the radius of the photosphere (in velocity space) must somehow increase with time.

In a single explosion, the ejecta naturally follow a Hubble law; however, multiple explosions can change the velocity profile of the ejecta. If two explosions occurred in short succession, one can produce the inverted velocity gradient seen in SN 2009ip. In this toy model, the photosphere would recede into the ejecta of the first explosion, but at some point the fastest-moving ejecta of the second explosion would overtake the photosphere, increasing the velocity. If there are no other explosions, the velocity of the absorption would decrease from there.

The photometric behavior of SN 2009ip is consistent with this picture. The first explosion would produce the fast rise to maximum. As noted by $\mathrm{S09}$, the timescale of the fading is much shorter than the timescales for many physical processes such as dust extinction 14 This behavior is very similar to that of SN $2000 \mathrm{ch}$, which brightened by $2.1 \mathrm{mag}$ in 9 days to maximum, then immediately faded by $3.4 \mathrm{mag}$ in 7 days, immediately followed by a $2.2 \mathrm{mag}$ rise in 4 days, after which the magnitude stayed relatively constant (Wagner et al. 2004). Spectra of SN 2000ch taken during the fading and on its plateau show no strong evidence for high-velocity ejecta, but the spectra may not be of high enough quality to see these features.

S09 hypothesized that rapid fading may have been caused by an optically thick shell being ejected after the first outburst. If this process did occur in SN 2009ip, then there are several implications: (1) the velocity of the absorption should eventually decrease, (2) the interaction of the ejecta from the two explosions could be a significant source of X-ray and radio emission, and (3) the X-rays might excite certain elements producing highexcitation lines such as He II in optical spectra. Our Xray limit of $L_{X}<4.8 \times 10^{38} \mathrm{ergs} \mathrm{s}^{-1}$ taken during the minimum is not particularly constraining. We do not detect any He II $\lambda 4686$ emission in the 4 or 24 day spectra; however, there is a low significance detection of a line consistent with He II $\lambda 4686$ emission in the 86 day spectrum. Additional spectroscopy is necessary to determine the late-time velocity gradient.

\section{CONCLUSIONS}

We have presented extensive UV, optical, and NIR data for two transients, SN 2009ip and UGC 2773 OT2009-1. Although these events appear to be similar phenomena (luminous outbursts of massive stars), the details of the events show that there are many differences. These differences provide examples of the diversity of such events.

A previous study of these events, $\mathrm{S} 09$, provided an initial analysis of the object. Although the two studies agree on many points, our interpretation of the entire

\footnotetext{
14 The calculation by (S09) for the time until dust formation for SN 2009ip assumes a velocity of $500 \mathrm{~km} \mathrm{~s}^{-1}$. Although the ejecta are moving much faster than this assumed value, they would need to have a velocity of $\gtrsim 20,000 \mathrm{kms}^{-1}$ to reach the sublimation radius at the time of fading.
} 
data set is somewhat different than that of S09. In particular, we agree that based on pre-outburst HST imaging, historical light curves, and outburst spectroscopy, the progenitors of SN 2009ip and UGC 2773 OT2009-1 are LBVs with masses of $\gtrsim 60$ and $\gtrsim 25 M_{\odot}$, respectively. We also agree that the spectra of the two events are significantly different, but consistent with known LBVs or LBV outbursts. While UGC 2773 OT2009-1 had a cooler spectrum with a forest of absorption lines reminiscent of a F-type supergiant (similar to $\mathrm{S}$ Dor in its high state), SN 2009ip had a hot spectrum and exhibited mainly $\mathrm{H}$ Balmer emission (similar to other LBV giant eruptions). The spectral characteristics (particularly [Ca II] emission) and circumstellar dust link UGC 2773 OT2009-1 to the lower-mass, dust-obscured progenitors of NGC 300 OT2008-1 and SN 2008S. We agree that the progenitors of these objects are all massive stars and may have many characteristics similar to those of the LBV class, which could extend the mass range for LBV-like activity to relatively low-mass stars.

However, there are distinct differences between the analyses of S09 and of that presented here. Specifically, the initial mass ranges for the progenitors are slightly different in the two studies, with S09 estimating $50-$ $80 M_{\odot}\left(\right.$ instead of $\left.\gtrsim 60 M_{\odot}\right)$ and $\gtrsim 20 M_{\odot}$ (instead of $\left.\gtrsim 25 M_{\odot}\right)$ for SN 2009ip and UGC 2773 OT2009-1, respectively. The differences lie in the conversion from $H S T$ filters to Bessell filters and the adapted color range for the progenitor of SN 2009ip, and the additional information provided by stars in the vicinity of the progenitor of UGC 2773 OT2009-1.

Our interpretation of the exact nature of UGC 2773 OT2009-1 differs from that of S09. While S09 contends that this object is a true giant eruption of an LBV, we question this assertion and propose that it may be the result of extreme S Dor variability.

While S09 found an NIR excess for UGC 2773 OT20091 , hypothesizing that there may be dust emission as it is vaporized, we find more conclusive evidence for this scenario through our NIR spectroscopy. The NIR spectrum is consistent with an additional blackbody with $T \approx 2100 \mathrm{~K}$, but is inconsistent with reddening by dust. The presence of this dust indicates that the initial mass estimate of the progenitor (based on optical HST imaging) is a lower limit, and that the true initial mass is likely much larger.

In addition to what was discussed by S09, we have also detected high-velocity absorption in the spectra of SN 2009ip, indicative of an explosion (as opposed to subsonic outburst). The absorption has an inverse velocity gradient suggesting multiple explosions in short succession. The rapid fading and brightening shortly after maximum brightness noted by (S09) is consistent with multiple explosions, where a second explosion ejects an optically thick shell that temporarily dims the object.

We also note the spectroscopic similarity of UGC 2773 OT2009-1 and SN 1994W, which Dessart et al. (2009) has previously suggested was not a true SN that destroyed its progenitor star.

SN 2009ip and UGC 2773 OT2009-1 are very different manifestations of a similar phenomenon: extreme brightening of massive stars. With these objects and similar events (such as $\eta$ Car, NGC 300 OT2008-1, and SNe 1961V, 1954J, 1997bs, 2000ch, 2002kg, and 2008S), we show that luminous outbursts of massive stars are very heterogeneous. Some of this diversity is likely linked to the instability that causes the eruption, while some is caused by the circumstellar environment. Additional observations of new massive star eruptions are necessary to determine the physical mechanisms of the eruptions, the content of the circumstellar environments, and whether the two are physically connected.

Facilities: $\quad$ ARC (TripleSpec), FMO:31in (FanCam), Gemini:Gillett (GMOS), HST (WFPC2), Magellan:Baade (IMACS), Magellan:Clay (MagE), MMT (Blue Channel), Swift (UVOT, XRT)

R.J.F. is supported by a Clay Fellowship. O.D.F. is grateful for support from NASA GSRP, ARCS, and VSGC. E.M.L. is supported in part by a Ford Foundation Predoctoral Fellowship.

We are indebted to the staffs at the APO, Gemini, Magellan, and MMT Observatories for their dedicated services. We thank K. Olsen and R. McDermid for obtaining some of the data presented in the paper. We thank R. Chornock and R. Kirshner for stimulating discussions about the transients.

Based on observations made with the NASA/ESA Hubble Space Telescope, obtained from the data archive at the Space Telescope Science Institute. STScI is operated by the Association of Universities for Research in Astronomy, Inc. under NASA contract NAS 5-26555. Based in part on observations obtained at the Gemini Observatory, which is operated by the Association of Universities for Research in Astronomy, Inc., under a cooperative agreement with the US National Science Foundation on behalf of the Gemini partnership: the NSF (United States), the Science and Technology Facilities Council (United Kingdom), the National Research Council (Canada), CONICYT (Chile), the Australian Research Council (Australia), Ministério da Ciência e Tecnologia (Brazil) and Ministerio de Ciencia, Tecnología e Innovación Productiva (Argentina); the 6.5 meter Magellan Telescopes located at Las Campanas Observatory, Chile; the MMT Observatory, a joint facility of the Smithsonian Institution and the University of Arizona; the Fan Mountain Observatory 0.8 meter telescope; and the Apache Point Observatory 3.5 meter telescope, which is owned and operated by the Astrophysical Research Consortium. We acknowledge the use of public data from the Swift data archive.

\section{REFERENCES}

Abbott, D. C., \& Conti, P. S. 1987, ARA\&A, 25, 113

Ball, L., Campbell-Wilson, D., Crawford, D. F., \& Turtle, A. J. 1995, ApJ, 453, 864

Berger, E., \& Foley, R. J. 2009, The Astronomer's Telegram, 2187, 1

Berger, E., Foley, R. J., \& Ivans, I. 2009a, The Astronomer's Telegram, 2184, 1

Berger, E., et al. 2009b, ApJ, 699, 1850

Blondin, S., et al. 2006, AJ, 131, 1648

Boles, T. 2009, Central Bureau Electronic Telegrams, 1931, 1

Bond, H. E., Bedin, L. R., Bonanos, A. Z., Humphreys, R. M., Monard, L. A. G. B., Prieto, J. L., \& Walter, F. M. 2009, ApJ, 695, L154

Chu, Y., Gruendl, R. A., Stockdale, C. J., Rupen, M. P., Cowan, J. J., \& Teare, S. W. 2004, AJ, 127, 2850 
Chugai, N. N., et al. 2004, MNRAS, 352, 1213

Cushing, M. C., Vacca, W. D., \& Rayner, J. T. 2004, PASP, 116, 362

Dessart, L., Hillier, D. J., Gezari, S., Basa, S., \& Matheson, T. 2009, MNRAS, 394, 21

Dolphin, A. E. 2000, PASP, 112, 1383

Filippenko, A. V., Barth, A. J., Bower, G. C., Ho, L. C., Stringfellow, G. S., Goodrich, R. W., \& Porter, A. C. 1995, AJ, 110,2261

Foley, R. J., et al. 2009, AJ, 138, 376

- 2003, PASP, 115, 1220

- 2006, ApJ, 645, 450

Frew, D. J. 2004, Journal of Astronomical Data, 10, 6

Garnavich, P., Jha, S., Kirshner, R., Calkins, M., \& Brown, W. 1999, IAU Circ., 7150, 1

Gogarten, S. M., Dalcanton, J. J., Murphy, J. W., Williams, B. F., Gilbert, K., \& Dolphin, A. 2009, ApJ, 703, 300

Goodrich, R. W., Stringfellow, G. S., Penrod, G. D., \& Filippenko, A. V. 1989, ApJ, 342, 908

Herter, T. L., et al. 2008, in Society of Photo-Optical Instrumentation Engineers (SPIE) Conference Series, Vol. 7014 Society of Photo-Optical Instrumentation Engineers (SPIE) Conference Series

Hook, I. M., Jørgensen, I., Allington-Smith, J. R., Davies, R. L., Metcalfe, N., Murowinski, R. G., \& Crampton, D. 2004, PASP, 116,425

Horne, K. 1986, PASP, 98, 609

Humphreys, R. M., \& Davidson, K. 1994, PASP, 106, 1025

Kanneganti, S., Park, C., Skrutskie, M. F., Wilson, J. C., Nelson, M. J., Smith, A. W., \& Lam, C. R. 2009, PASP, 121, 885

Kelson, D. D. 2003, PASP, 115, 688

Kotak, R., et al. 2009, ApJ, 704, 306

Leonard, D. C., Filippenko, A. V., Barth, A. J., \& Matheson, T. 2000, ApJ, 536, 239

Li, W., Smith, N., Miller, A. A., \& Filippenko, A. V. 2009, The Astronomer's Telegram, 2212, 1

Marshall, J. L., et al. 2008, in Society of Photo-Optical Instrumentation Engineers (SPIE) Conference Series, Vol. 7014 Society of Photo-Optical Instrumentation Engineers (SPIE) Conference Series

Massey, P. 2000, PASP, 112, 144

Maund, J. R., et al. 2006, MNRAS, 369, 390

Maza, J., et al. 2009, Central Bureau Electronic Telegrams, 1928, 1

Miller, A. A., Li, W., Nugent, P. E., Bloom, J. S., Filippenko, A. V., \& Merritt, A. T. 2009, The Astronomer's Telegram, 2183, 1

Mould, J. R., et al. 2000, ApJ, 529, 786

Prieto, J. L., et al. 2008, ApJ, 681, L9
Schaller, G., Schaerer, D., Meynet, G., \& Maeder, A. 1992, A\&AS, 96, 269

Schlegel, D. J., Finkbeiner, D. P., \& Davis, M. 1998, ApJ, 500, 525

Schmidt, G. D., Weymann, R. J., \& Foltz, C. B. 1989, PASP, 101, 713

Schultz, G. V., \& Wiemer, W. 1975, A\&A, 43, 133

Smith, N. 2008, Nature, 455, 201

Smith, N., Chornock, R., Silverman, J. M., Filippenko, A. V., \& Foley, R. J. 2009a, ArXiv e-prints, 0906.2200

Smith, N., Foley, R. J., \& Filippenko, A. V. 2008a, ApJ, 680, 568

Smith, N., Ganeshalingam, M., Li, W., Chornock, R., Steele, T. N., Silverman, J. M., Filippenko, A. V., \& Mobberley, M. P. 2008b, ArXiv e-prints, 0811.3929

Smith, N., Gehrz, R. D., Hinz, P. M., Hoffmann, W. F., Hora, J. L., Mamajek, E. E., \& Meyer, M. R. 2003, AJ, 125, 1458

Smith, N., Humphreys, R. M., \& Gehrz, R. D. 2001, PASP, 113, 692

Smith, N., et al. 2009b, ArXiv e-prints, 0909.4792

Soderberg, A. M., et al. 2008, Nature, 453, 469

Sollerman, J., Cumming, R. J., \& Lundqvist, P. 1998, ApJ, 493, 933

Stockdale, C. J., Rupen, M. P., Cowan, J. J., Chu, Y., \& Jones, S. S. 2001, AJ, 122, 283

Sugerman, B., Meixner, M., Fabbri, J., \& Barlow, M. 2004, IAU Circ., 8442, 2

Van Dyk, S. D., Filippenko, A. V., Chornock, R., Li, W., \& Challis, P. M. 2005, PASP, 117, 553

Van Dyk, S. D., Filippenko, A. V., \& Li, W. 2002, PASP, 114, 700

Van Dyk, S. D., Li, W., Filippenko, A. V., Humphreys, R. M., Chornock, R., Foley, R., \& Challis, P. M. 2006, ArXiv Astrophysics e-prints

Van Dyk, S. D., Peng, C. Y., King, J. Y., Filippenko, A. V., Treffers, R. R., Li, W., \& Richmond, M. W. 2000, PASP, 112, 1532

van Dyk, S. D., Weiler, K. W., Sramek, R. A., Schlegel, E. M., Filippenko, A. V., Panagia, N., \& Leibundgut, B. 1996, AJ, 111,1271

van Genderen, A. M. 2001, A\&A, 366, 508

Wade, R. A., \& Horne, K. 1988, ApJ, 324, 411

Wagner, R. M., et al. 2004, PASP, 116, 326

Weis, K., \& Bomans, D. J. 2005, A\&A, 429, L13

Wilson, J. C., et al. 2004, in Society of Photo-Optical Instrumentation Engineers (SPIE) Conference Series, Vol. 5492 , Society of Photo-Optical Instrumentation Engineers (SPIE)

Conference Series, ed. A. F. M. Moorwood \& M. Iye, 1295-1305 